\title{
An Experimental and Theoretical Study of the Reactivity of Selected Catecholamines and Their Precursors Towards Ascorbyl Radical
}

\author{
Dušan Dimić $^{1}$, Đura Nakarada ${ }^{1}$, Miloš Mojović ${ }^{1}$, Jasmina Dimitrić Marković ${ }^{*}$ \\ ${ }^{1}$ Faculty of Physical Chemistry, University of Belgrade, Studentski trg 12-16, 11000 Belgrade, \\ Serbia \\ e-mail: ddimic@ffh.bg.ac.rs, djura@ffh.bg.ac.rs, milos@ffh.bg.ac.rs, markovich@ffh.bg.ac. \\ *corresponding author
}

\begin{abstract}
Ascorbyl radical is often used as a biomarker of oxidative stress in human organism. It is a product of the antioxidant activity of ascorbic acid and it is not expected to react further with biologically important molecules. For the first time, the reactivity of catecholamines and their precursors was investigated theoretically and experimentally towards ascorbyl radical and the main structural parameters governing activity were analyzed. It was shown that catechol moiety plays an important role, which classifies norepinephrine and 3,4-dihydroxyphenylacetic acid as the most reactive when compared to homovanillic acid, vanillylmandelic acid, and octopamine. DFT methods have been employed to determine the most probable mechanism of the reaction. Based on the change in reaction enthalpy it was concluded that Hydrogen Atom Transfer (HAT) is a preferred mechanism both in water and pentyl ethanoate. The stabilization interactions in ascorbic acid, its anion and radical are carefully analyzed in order to understand the preferability of the mentioned mechanism. The stability of the ascorbyl radical is explained in detail. The results prove that ascorbyl radical is not just a product of antioxidant activity, but also a potentially harmful species in an organism.
\end{abstract}

Keywords: EPR, ascorbyl radical, catecholamines, antiradical activity

\section{Introduction}

Free radicals are chemical species characterized by one or multiple unpaired electrons which govern their high reactivity. They are normally produced by aerobic metabolism and many free radicals have important biological functions. Once their concentration is higher than usual the balance between oxidant and antioxidant statuses is shifted (Sies, 1991; Halliwell and Gutteridge, 1999). In such a case organism fights against reactive species by activation of internal and external antioxidants.

The pathology of neurological disorders, such as Huntington's, Alzheimer's and Parkinson's, includes the increased concentration of reactive oxygen species (ROS) (Halliwell and Gutteridge, 1999). This can be expected because more than $20 \%$ of oxygen is used in the brain (D. Dimić et al., 2017a). Some of the effects of ROS are lipid peroxidation, mitochondrial dysfunction, increase in the concentration of redox metals, malondialdehyde, and vitamin $\mathrm{E}$, but also decrease in the concentration of neurotransmitters (Andersen, 2004). The reactivity of 
neurotransmitters has been shown experimentally and theoretically towards various radicals: superoxide anion (Kladna et al., 2013), chlorinated methylperoxy (D. S. Dimić et al., 2017), singlet oxygen (Gülçin, 2009; Shimizu et al., 2010), hydroxyl (Kładna et al., 2012), 2,2diphenyl-1-picrylhydrazyl (Sârbu and Casoni, 2013; D. Dimić et al., 2017a) and 2,2'-azinobis(3-ethylbenzothiazoline-6-sulphonic acid) (Dimic et al., 2019) radicals.

The molecules chosen for this investigation include norepinephrine, 3,4dihydroxylphenylacetic acid (DOPAC), vanillylmandelic acid (VMA), homovanillic acid (HVA) and octopamine (Figure 1). Norepinephrine is a hormone in the central nervous system involved in movement control, mood modulation and other important functions (D. S. Dimić et al., 2017). DOPAC is the most important metabolite of dopamine in the brain (Laranjinha and Cadenas, 2002; Lopes Jesus et al., 2015). Both of these molecules are characterized by the presence of catechol moiety in their structure which is an important structural parameter of good antioxidants (Dimitrić Marković et al., 2014; Petrović et al., 2015). Vanillylmandelic acid is an important metabolite of norepinephrine and epinephrine that is routinely screened when disorder in neurotransmitter metabolism is suspected (Armstrong, McMillan and Shaw, 1957; Dimić et al., 2018). HVA is another metabolite of dopamine that is a marker of dopamine concentration (Samsonowicz et al., 2014). The latter two molecules have one hydroxy and one methoxy group attached directly to an aromatic ring. Octopamine is a structural analog of norepinephrine with one hydroxy group and acts as a neurotransmitter in invertebrates (Copeland and Robertson, 1982).

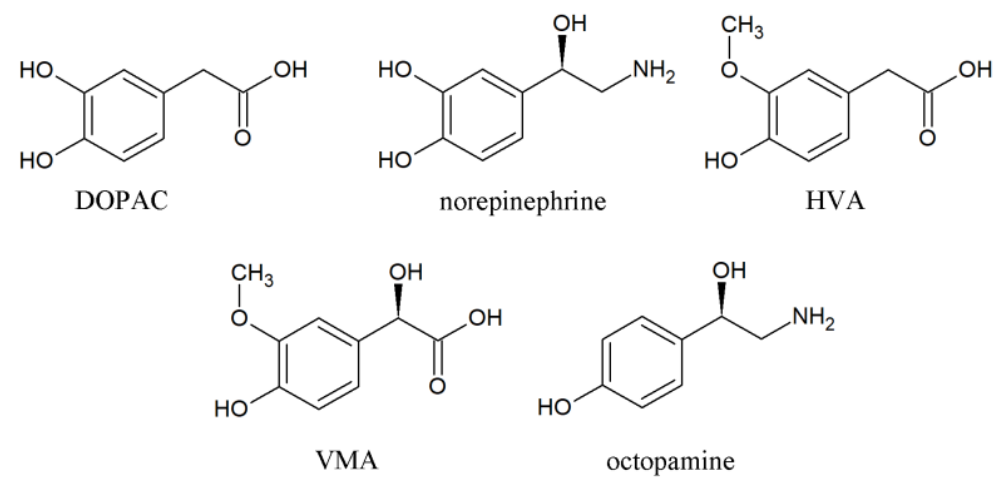

Fig. 1. Structures of selected catecholamines and their precursors.

Ascorbyl radical is produced in a humane organism when ascorbic acid reacts with free radicals. Ascorbic acid can behave as antioxidant or pro-oxidant in the presence of different enzymes and catalysts (Pietri et al., 1990; Roginsky and Stegmann, 1994). Ascorbyl radical has a characteristic EPR spectrum, and it is used as a marker of oxidative stress (González, Aguiar and Malanga, 2013). This is a very stable radical species therefore is it suitable for measurement of the presence of reactive species in tissues and living organisms. The concentration of ascorbyl radical increases in the presence of pesticides, the intoxication of bacterial toxins and hydrogen-peroxide. It was shown that the radical activity of polyphenols on ascorbyl radical depends on molecular weight, functional groups, and the presence of polysaccharides (Satoh and Sakagami, 1996).

There are three commonly investigated mechanisms of reduction of reactive species which differ in a number of steps or transferring particles between antioxidant and free radical (Leopoldini, Russo and Toscano, 2011; Galano et al., 2016; Marković et al., 2016; D. Dimić et 
al., 2017a; Tošović et al., 2017). Hydrogen atom transfer (HAT) is a single step mechanism that includes a transfer of hydrogen atom from antioxidants to free radicals which leads to the formation of more stable radicals from antioxidants (Eq. 1).

$$
\mathrm{ArOH}+\mathrm{R}^{\bullet} \rightarrow \mathrm{ArO}^{\bullet}+\mathrm{RH}
$$

The second mechanism is the Sequential Proton Loss Electron Transfer (SPLET) which consists of proton transfer followed by electron transfer. The products in the first step are anion of antioxidant species and proton (Eq. 2). The anion afterward reacts with radical and electron and anionic species from radical is formed (Eq. 3). In the last step, the protonation of anionic species occurs (Eq. 4).

$$
\begin{gathered}
\mathrm{ArOH} \rightarrow \mathrm{ArO}^{-}+H^{+} \\
\mathrm{ArO}^{-}+\mathrm{R}^{\bullet} \rightarrow \mathrm{ArO} \mathrm{O}^{\bullet}+R^{-} \\
R^{-}+H^{+} \rightarrow R H
\end{gathered}
$$

The Single Electron Transfer - Proton Transfer (SET-PT) is also a two-step mechanism that includes electron transfer and formation of radical cation of antioxidant and anion of radical species (Eq. 5). In the second step, a proton is exchanged between these two species (Eq. 6).

$$
\begin{aligned}
& \mathrm{ArOH}+\mathrm{R}^{\bullet} \rightarrow \mathrm{ArOH}^{\bullet+}+R^{-} \\
& \mathrm{ArOH}^{\bullet+}+R^{-} \rightarrow \mathrm{ArO}^{\bullet}+\mathrm{RH}
\end{aligned}
$$

This contribution gives an experimental and theoretical consideration of the reaction between selected catecholamines and their precursors with ascorbyl radicals. The experimental and theoretical methods are explained in the next section. Based on the EPR spectra the reactivity of the mentioned scavengers is calculated and the most important structural parameters outlined. Three of the most common radical scavenging processes examined by the change in enthalpy of reaction are used to determine the preferred mechanism. The comparison of experimental and theoretical results allows, for the first time in literature, the construction of the overall picture of reactions between biologically important molecules with ascorbyl radicals.

\section{Methods}

\subsection{Experimental method}

The investigated compounds, norepinephrine hydrochloride, 3,4-dihydroxyphenylacetic acid (DOPAC), octopamine hydrochloride, homovanillic acid, vanillylmandelic acid, ascorbic acid, were purchased from Sigma Aldrich Co. All of the chemicals were of reagent grade.

The instrument used in experiments was the Bruker Elexsys-II EPR spectrometer. The ascorbyl radical was generated from ascorbic acid in $0.1 \mathrm{M}$ phosphate buffer saline (PBS) at $\mathrm{pH}=7.6$. The starting solution of ascorbic acid was $10 \mathrm{mM}$, the same as a stock solution of all of the investigated molecules. The final solution contained $11 \mu \mathrm{L}$ of ascorbic acid, $15 \mu \mathrm{L}$ of catecholamines and their precursors and $2 \mu \mathrm{L}$ of a buffer. The signal of ascorbyl radical was monitored between the $4^{\text {th }}$ and $6^{\text {th }}$ minutes since the start of the reaction. The antiradical activity (AA) of these molecules was calculated as the relative decrease in the height of probe with (Iox) and without radical scavenger ( $\mathrm{I}_{\mathrm{CONT}}$ ) by the following formula:

$$
A A=\left(1-\frac{I_{O X}}{I_{C O N T}}\right) \cdot 100 \%
$$




\subsection{Computational methods}

The structures of investigated molecules were optimized in the Gaussian program package (Frisch et al., 2009). The calculations included neutral molecules of catecholamines and their precursors, as well as their radicals, anions, and radical cations at M06-2X (Zhao and Truhlar, 2007) level of theory with 6-311++G(d,p) (Dunning, 1989) basis set. The optimization was performed without any geometrical constraints. The absence of imaginary frequencies proved that local and global minima were found. In order to encounter for the possible differences in the mechanism of antiradical activity in aqueous and lipid environments, two solvents were investigated - water and pentyl ethanoate. These two solvents were modeled by the implemented SMD model (Marenich, Cramer and Truhlar, 2009).

The thermodynamic parameters governing the mentioned mechanisms can be calculated as the change in the enthalpy of reaction between radicals and radical scavengers. The thermodynamic parameters determine the preferability of selected mechanisms (Marković et al., 2015; D. S. Dimić et al., 2017; Milenković et al., 2017; Dimic et al., 2019). The bond dissociation enthalpy $\left(\Delta H_{\mathrm{BDE}}\right)$, proton affinity $\left(\Delta H_{\mathrm{PA}}\right)$, electron transfer enthalpy $\left(\Delta H_{\mathrm{ETE}}\right)$, ionization potential $\left(\Delta H_{\mathrm{IP}}\right)$ and proton dissociation enthalpy $\left(\Delta H_{\mathrm{PDE}}\right)$ are parameters associated with mentioned mechanisms.

$$
\begin{gathered}
\Delta H_{B D E}=H\left(A r O^{\bullet}\right)+H(R H)-H(A r O H)-H\left(R^{\bullet}\right) \\
\Delta H_{P A}=H\left(A r O^{-}\right)+H(R H)-H(A r O H)-H\left(R^{-}\right) \\
\Delta H_{E T E}=H\left(A r O^{\bullet}\right)+H\left(R^{-}\right)-H\left(A r O^{-}\right)-H\left(R^{\bullet}\right) \\
\Delta H_{I P}=H\left(A r O H^{+}\right)+H\left(R^{-}\right)-H(A r O H)-H\left(R^{\bullet}\right) \\
\Delta H_{B D E}=H\left(A r O^{\bullet}\right)+H(R H)-H\left(A r O H^{+\bullet}\right)-H\left(R^{-}\right)
\end{gathered}
$$

\section{Results and Discussion}

Prior to the investigation of reactivity of catecholamines and their precursors, the stability of formed ascorbyl radical is examined for the expected time period of reaction (between $4^{\text {th }}$ and $6^{\text {th }}$ minute after the start of reaction). Figure 2a gives the EPR spectrum of ascorbyl radical in the $4^{\text {th }}$ and $6^{\text {th }}$ minute after the generation of radicals. On the other hand, the signal of ascorbyl radical is recorded in $4^{\text {th }}, 6^{\text {th }}$ and $8^{\text {th }}$ minute after the addition of norepinephrine in order to determine the reaction time before the height stabilizes. These results are given in Figure $2 \mathrm{~b}$. 


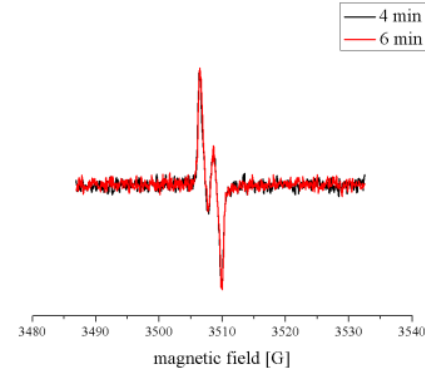

a)

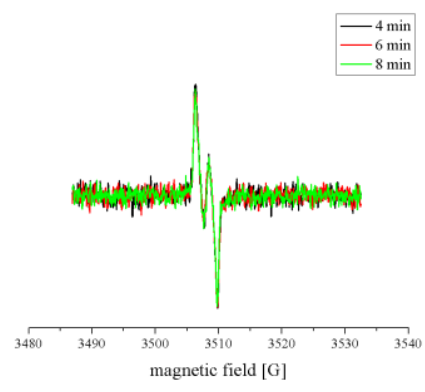

b)

Fig. 2. The ascorbyl radical signal a) 4 and 6 minutes after the formation and b) 4,6 and 8 minutes after the addition of norepinephrine

As can be seen in Figure 1a, the signal of formed radical was constant in the observed time range. The EPR signal height after the addition of norepinephrine, final concentration $\left(5 \times 10^{-3}\right.$ M) was constant after the $4^{\text {th }}$ minute. This allowed the estimation of reaction time which was lower than 4 minutes, therefore it was decided to take the peak height after $4^{\text {th }}$ minute as the measure of the reactivity of selected catecholamines and their precursors. The experimental results for the reactivity of selected catecholamines and their precursors are given in Figure 3.

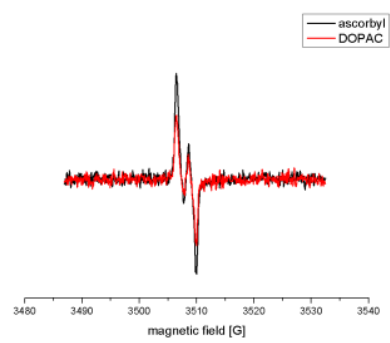

a)

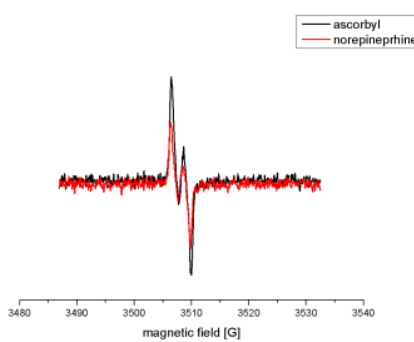

b)

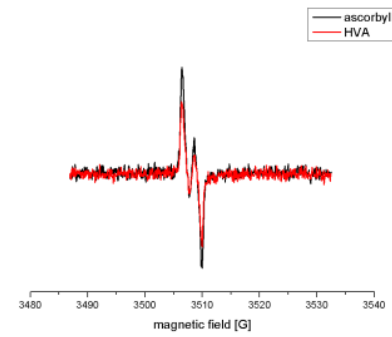

c)

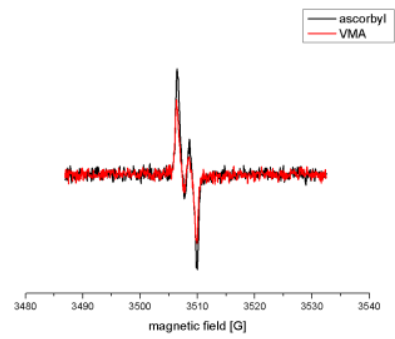

d)

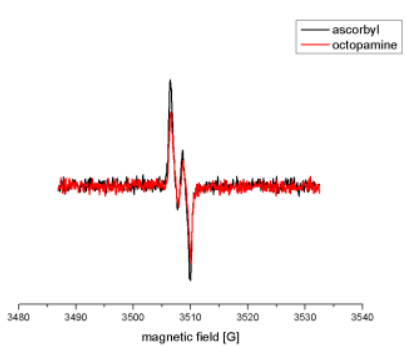

e)

Fig. 3 The ascorbyl signal before and after the addition of a) DOPAC, b) norepinephrine, c) HVA, d) VMA and e) octopamine (final concentration $5 \times 10^{-3} \mathrm{M}$ ).

It is obvious from Figure 3 that the addition of selected substances lowers the intensity of the signal. The percentage of reduction for norepinephrine and DOPAC is $35 \%$, for DOPAC, for HVA and VMA is $27 \%$ and for octopamine is $24 \%$. These results show that the reactivity of investigated molecules towards ascorbyl radical are similar, although some subgroups can be made. This is an interesting result because the percentages of reduction of other radicals were much higher (D. Dimić et al., 2017a; Dimic et al., 2019). The differences in reactivity can be 
attributed to different structural parameters. Catechol moiety is an important structural parameter, as previously shown (Sârbu and Casoni, 2013). Norepinephrine and DOPAC have this structural parameter and the measured activity is the highest among investigated substances. On the other hand, the ending group of the aliphatic chain can contribute to the total activity, as shown in the case of DPPH' (D. Dimić et al., 2017a). In the case of ascorbyl radical, there is no difference in reactivity of molecules with an amino group (norepinephrine) and a carboxyl group (DOPAC). One of the reasons is that the measurements were performed in a buffered solution with $\mathrm{pH}=7.6$, which means that DOPAC is more than $99.9 \%$ deprotonated. HVA and VMA also have the same percentage of reduction. These two molecules differ only in the hydroxy group attached to the aliphatic chain of VMA. This same group is present in the structure of norepinephrine, and, when reactivities are compared, it can be concluded that it does not represent a significant position. The reactivity of HVA and VMA is lower due to the fact that one of the hydroxy groups of an aromatic ring is exchanged with methoxy group, which influences the hydrogen bond strength in formed radicals and their overall stability. Octopamine has the lowest reactivity towards ascorbyl radicals. This is again a consequence of the number and nature of substituents on the aromatic ring.

The possible reaction pathways, as already dissed in the Introduction, are presented in Figure 4. The reduction of ascorbyl radical back to ascorbic acid can proceed through several mechanisms. The transfer of hydrogen atom is a one-step mechanism with a direct reduction of radicals. The second mechanism represents both SPLET and SET-PT because the radical species first accepts an electron and afterward a proton. Table 1 gives values of thermodynamic parameters, calculated by Eqs. 8-12 both in water and pentyl ethanoate.

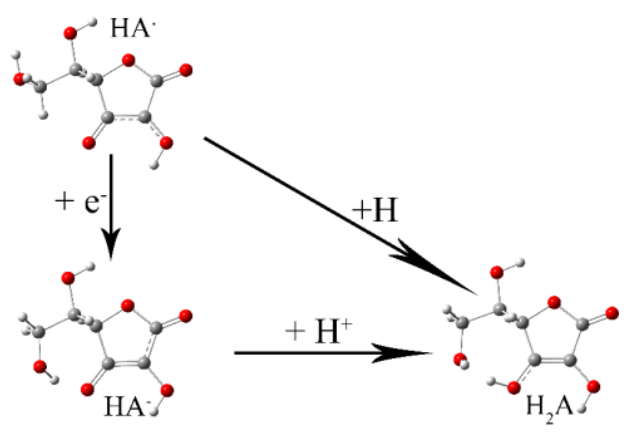

Fig. 4. Possible reaction pathways for the reduction of ascorbyl radical to ascorbic acid.

\begin{tabular}{c|c|c|c|c|c|c|c|c|c|c}
\hline \multirow{2}{*}{ Molecule } & \multicolumn{4}{|c|}{ Aqueous } & \multicolumn{4}{c}{ Pentyl ethanoate } \\
\cline { 2 - 12 } & HAT & \multicolumn{2}{|c|}{ SET-PT } & \multicolumn{2}{c|}{ SPLET } & HAT & \multicolumn{2}{c}{ SET-PT } & \multicolumn{2}{c}{ SPLET } \\
\cline { 2 - 11 } & $\Delta \mathrm{H}_{\mathrm{BDE}}$ & $\Delta \mathrm{H}_{\mathrm{IP}}$ & $\Delta \mathrm{H}_{\mathrm{PDE}}$ & $\Delta \mathrm{H}_{\mathrm{PA}}$ & $\Delta \mathrm{H}_{\mathrm{ETE}}$ & $\Delta \mathrm{H}_{\mathrm{BDE}}$ & $\Delta \mathrm{H}_{\mathrm{IP}}$ & $\Delta \mathrm{H}_{\mathrm{PDE}}$ & $\Delta \mathrm{H}_{\mathrm{PA}}$ & $\Delta \mathrm{H}_{\mathrm{ETE}}$ \\
\hline \multirow{2}{*}{ DOPAC } & 5,9 & 74,7 & $-68,8$ & 46,8 & $-40,9$ & 1,4 & 172,9 & $-171,4$ & 66,5 & $-65,1$ \\
Norepinephrine & 4,4 & 71,2 & $-66,8$ & 48,0 & $-43,6$ & $-0,02$ & 162,5 & $-162,5$ & 71,1 & $-71,1$ \\
VMA & 17,7 & 76,1 & $-58,4$ & 57,1 & $-39,4$ & 28,3 & 166,8 & $-138,3$ & 101,2 & $-72,9$ \\
HVA & 12,9 & 69,7 & $-56,8$ & 62,4 & $-49,6$ & 25,4 & 160,2 & $-134,8$ & 111,8 & $-86,5$ \\
Octopamine & 30,5 & 93,5 & $-63,5$ & 61,3 & $-30,8$ & 30,5 & 182.3 & -151.8 & 97,0 & $-66,6$ \\
\hline
\end{tabular}

Table 1. The values of the enthalpy change for reaction between catecholamines and their precursors and ascorbyl radical for different reaction mechanisms (in $\mathrm{kJ} \mathrm{mol}^{-1}$ ) 
The results presented in Table 1 show that thermodynamic parameters are also dependent on the present structural moieties. The values are positive for all molecules in water and for DOPAC, VMA, HVA, and octopamine in pentyl ethanoate. When values for $\Delta H_{\mathrm{BDE}}$ are compared it can be concluded that this parameter reflects well the experimentally obtained reactivity order. The lowest values for $\Delta H_{\mathrm{BDE}}$ are calculated for norepinephrine $\left(4.4 \mathrm{~kJ} \mathrm{~mol}^{-1}\right)$ and DOPAC $\left(5.9 \mathrm{~kJ} \mathrm{~mol}^{-1}\right)$ and this is a consequence of strong hydrogen bond formed within catechol moiety. When methoxy group is present this interaction is much weaker and values for $\Delta H_{\mathrm{BDE}}$ are 17.7 and $12.9 \mathrm{~kJ} \mathrm{~mol}^{-1}$ for VMA and HVA, respectively. Approximately two times higher value is obtained for octopamine due to the fact that no stabilization interaction is observed. The preferred mechanism is characterized by the lowest value of the enthalpy change. When parameters for the first steps of each mechanism are compared for DOPAC, their values are 5.9, 74.7 and $46.8 \mathrm{~kJ} \mathrm{~mol}^{-1}$ for $\Delta H_{\mathrm{BDE}}, \Delta H_{\mathrm{IP}}$, and $\Delta H_{\mathrm{PA}}$, respectively. It is very common that SET-PT is the least probable mechanism in polar solvents (Marković et al., 2014; Filipović et al., 2015; D. Dimić et al., 2017b; Milenković et al., 2017). Both the second steps of SET-PT and SPLET mechanisms are negative because stable species are formed. The same trend can be seen for other investigated substances. When values for specific parameters are compared it can be concluded that not all of them uniformly change with structure and not all of them reflect well the experimental results. Values within $6 \mathrm{~kJ} \mathrm{~mol}^{-1}$ are obtained for $\Delta H_{\mathrm{IP}}$ for norepinephrine, DOPAC, HVA, and VMA. On the other hand, $\Delta H_{\mathrm{PA}}$ values are similar for norepinephrine and DOPAC in one group and in second for HVA, VMA, and octopamine. These values indicate that HAT is the most probable mechanism. The values of thermodynamic parameters in pentyl ethanoate are higher for all enthalpy changes except for $\Delta H_{\mathrm{BDE}}$ of norepinephrine, DOPAC and octopamine. In non-polar solvents, the values of $\Delta H_{\mathrm{BDE}}$ are much closer for HVA, VMA, and octopamine because polar groups of these molecules don't form significant interactions with solvent molecules and intramolecular interaction are weak. The values for $\Delta H_{\mathrm{IP}}$ and $\Delta H_{\mathrm{PA}}$ are more than two times higher in pentyl ethanoate than in water because the formed ionic species are not stabilized by interactions with the solvent. Again, the dominant mechanism is the HAT for all investigated molecules. This proves that the reaction between biologically important molecules and ascorbyl radicals is possible both in aqueous media and pentyl ethanoate.

The stabilization of the structure of antioxidants because of the hydrogen bond formation can be discussed based on the stabilization interactions. The results for these interactions are presented in Table 2 .

\begin{tabular}{c|c|c|c}
\hline \multirow{2}{*}{ Molecule } & \multicolumn{3}{|c}{ Aqueous } \\
\cline { 2 - 4 } & Neutral & Anionic & Radical \\
\hline DOPAC & 3.14 & 1.46 & 2.26 \\
Norepinephrine & 3.43 & 1.84 & 2.29 \\
VMA & 5.30 & 2.18 & $/$ \\
HVA & 5.26 & 2.17 & $/$ \\
Octopamine & $/$ & $/$ & $/$ \\
\hline
\end{tabular}

Table 2. The stabilization interactions in neurotransmitters and their precursors in the neutral, anionic and radical form (in $\mathrm{kJ} \mathrm{mol}^{-1}$ ).

The stabilization interaction is observed in DOPAC, norepinephrine, VMA, and HVA. The strongest hydrogen bonds are observed for neutral forms of VMA and HVA because of the charge distribution and electronegativity of the oxygen atom in para-positon. The interaction in 
anionic form is two times weaker for all investigated species. This leads to the lower stability of molecules in anionic form. The stabilization interaction in radical species is higher for DOPAC and norepinephrine than in anionic species. These results also contribute to the previous conclusion that the HAT mechanism is the dominant one in the investigated systems. The preferability of mechanism, therefore, depends both on the stabilization interactions in the starting molecules and the final products.

When the structure of ascorbic acid is analyzed it can be concluded that various intramolecular interactions stabilize the structure. This might be the reason why some of the mechanisms are preferred over others. The structure of ascorbic acid, its radical and anion are given in Figure 4. Ascorbic acid has four $\mathrm{OH}$ groups, three of which could possibly form intramolecular hydrogen bonds. On the other side anion and radical have three $\mathrm{OH}$ groups but the number of possible intramolecular hydrogen bonds remains the same. The results of the Second-order perturbation theory (SOPT) are presented in Table 3 so that the quantitative discussion of the bond strengths can be performed. The enumeration of oxygen atoms is given in Figure 5.

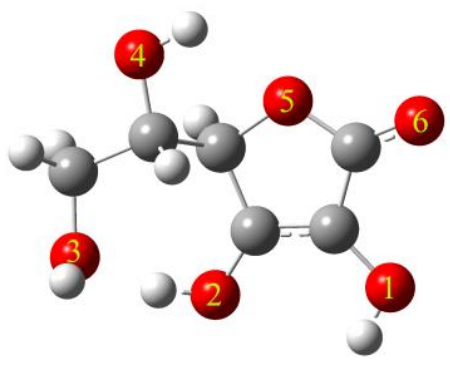

Fig. 5. Enumeration of the oxygen atoms important for intramolecular stabilization interaction.

\begin{tabular}{|c|c|c|c|c|c|c|c|c|c|}
\hline \multirow[b]{2}{*}{ Molecule } & \multicolumn{4}{|c|}{ Aqueous } & \multicolumn{5}{|c|}{ Oxygen atom charges } \\
\hline & Interaction & $\begin{array}{c}\text { Donor } \\
\text { atom } \\
\text { occupancy }\end{array}$ & $\begin{array}{c}\text { Acceptor } \\
\text { atom } \\
\text { occupancy }\end{array}$ & $\begin{array}{c}\text { Stabilization } \\
\text { interaction } \\
\text { energy }\end{array}$ & $\mathrm{O} 1$ & $\mathrm{O} 2$ & $\mathrm{O} 3$ & $\mathrm{O} 4$ & O5 \\
\hline $\begin{array}{l}\text { Ascorbic } \\
\text { acid }\end{array}$ & $\mathrm{O} 2-\mathrm{H}-\mathrm{O} 3$ & 1.94 & 0.05 & 86.73 & -0.68 & -0.71 & -0.75 & -0.78 & -0.59 \\
\hline $\begin{array}{l}\text { Ascorbic } \\
\text { acid anion }\end{array}$ & $\begin{array}{l}\text { O5-H-O4 } \\
\text { O3-H-O2 }\end{array}$ & $\begin{array}{l}1.97 \\
1.66\end{array}$ & $\begin{array}{l}0.01 \\
0.05\end{array}$ & $\begin{array}{c}2.67 \\
96.14\end{array}$ & -0.72 & -0.81 & -0.80 & -0.77 & -0.62 \\
\hline $\begin{array}{c}\text { Ascorbyl } \\
\text { radical }\end{array}$ & l & l & l & l & -0.59 & -0.55 & -0.76 & -0.76 & -0.57 \\
\hline
\end{tabular}

Table 3. The important stabilization interactions for stabilization of structure of ascorbic acid, its anion and radical (in $\mathrm{kJ} \mathrm{mol}^{-1}$ ) and the natural charges on oxygen atoms $\mathrm{O} 1$ to $\mathrm{O} 5$ (in $e$ ).

Interestingly, the results of SOPT showed only the limited number of interactions for the investigated systems for energies over the threshold value of $2.1 \mathrm{~kJ} \mathrm{~mol}^{-1}$. The strongest stabilization interaction including oxygen atoms is between $\mathrm{O} 2$ and $\mathrm{O} 3$ with the energy of 86.76 $\mathrm{kJ} \mathrm{mol}^{-1}$. Once the structure is deprotonated the reversed stabilization interaction is formed with $\mathrm{O} 3$ now being the hydrogen atom donor with the energy of $96.14 \mathrm{~kJ} \mathrm{~mol}^{-1}$. This bond is stronger due to the fact that negative charge is concentrated on $\mathrm{O} 2$. In radical, this bond is not formed because there is a rotation of the aliphatic chain which contains O3 (Figure 4). The natural 
charges on oxygen atoms are also given in Table 3. When charges in anion are compared with neutral molecule it can be concluded that the charge is delocalized over the whole structure because the increase in negative charge is seen on atoms $\mathrm{O} 1, \mathrm{O} 2, \mathrm{O} 3$ and $\mathrm{O} 5$. Because of this, the hydrogen bond between $\mathrm{O} 2$ and $\mathrm{O} 3$ is stronger. Once the radical is formed, the total negative charge is lowered on atoms $\mathrm{O} 2$ and $\mathrm{O} 3$, which means that the loss of electron occurs from position $\mathrm{O} 2$ but it is again delocalized over the furan ring. Due to the electron loss, the electronegativity of atoms is lowered which leads to the decrease in stabilization interaction strength as shown in Table 3.

One of the important questions raised in this study is the relative stability of the ascorbyl radical. Although it was shown that this species can react with biologically important molecules like neurotransmitters and their precursors, ascorbic acid still remains one of the most important antioxidants in the human organism and the first line of defense against oxidative stress. In order to access the stability of formed radicals, the stabilization interactions other than hydrogen bonds and spin delocalization are examined because the hydrogen bonds are not present in the structure, as discussed in the previous paragraph. Figure 6 gives the spin density for the ascorbyl radical.

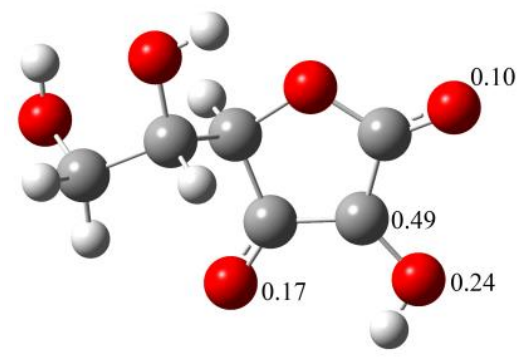

Fig. 6. Spin density delocalization in ascorbyl radical.

As can be seen in Figure 6 once the radical is formed the spin density is delocalized over the ring, with the most density being located on oxygen atoms and carbon atom attached to O1. The aliphatic chain does not contain a significant amount of spin density. The most important stabilization interactions include $\pi(\mathrm{C}-\mathrm{C}) \rightarrow \pi^{*}(\mathrm{C}-\mathrm{O}), \quad \mathrm{LP}(\mathrm{O} 5) \rightarrow \pi(\mathrm{C}-\mathrm{O} 6), \quad \mathrm{LP}(\mathrm{O} 6) \rightarrow \pi(\mathrm{C}-\mathrm{C})$, $\mathrm{LP}(\mathrm{O} 6) \rightarrow \pi^{*}(\mathrm{C}-\mathrm{O})$ and $\mathrm{LP}(\mathrm{O} 2) \rightarrow \pi^{*}(\mathrm{C}-\mathrm{C})$, all of which have interaction energy higher than 50 $\mathrm{kJ} \mathrm{mol}^{-1}$. Besides the stabilizations that usually exist in ring structures originating from the overlap between two $\pi$ orbitals, oxygen atoms also influence the structure through interactions between lone pair and $\pi$ orbitals. Therefore it can be concluded that the stability of investigated radicals is a consequence of the stabilization interactions, spin and charge delocalization.

\section{Conclusions}

The reactivity of norepinephrine, 3,4-dihidroxyphenylacetic acid (DOPAC), homovanillic acid (HVA), vanylilmandelic acid (VMA) and octopamine towards ascorbyl radical was investigated experimentally and theoretically. Based on the EPR spectroscopy measurements it was concluded that the same concentrations of norepinephrine and DOPAC reduced $35 \%$ of present radical, while HVA and VMA reduced $27 \%$. The lowest reduction $(24 \%)$ was observed for octopamine. The importance of the intramolecular hydrogen bonds in formed radicals is proven because catechol moiety is the most important structural parameter governing the activity. The most probable mechanism was determined based on the change of enthalpy of reaction. Out of 
the three most common mechanisms, the Hydrogen Atom Transfer (HAT) requires the lowest amount of energy in water and pentyl ethanoate. The values of thermodynamic parameters also reflect well the importance of catechol moiety. The Second-order perturbation theory results proved that stabilization interaction within catechol moiety is formed. The ascorbic acid, its radical and anion also show significant delocalization of charge and strong stabilization interactions within the structure. Once the radical is formed the spin density is delocalized over oxygen and carbon atoms of furan ring which leads to the relative stability of this species. Further research is needed, but this is one of the first attempts to prove that ascorbyl radicals can react with biologically important molecules after the reduction of ascorbic acid by free radicals.

Acknowledgments: This work was supported by the Serbian Ministry of Education, Science and Technological Development (451-03-68/2020-14/200146).

\section{References}

Andersen, J. K. (2004) 'Oxidative stress in neurodegeneration: cause or consequence?', Nature Reviews Neuroscience, 10(7), pp. S18-S25. doi: 10.1038/nrn1434.

Armstrong, M. D., McMillan, A. and Shaw, K. N. (1957) '3-Methoxy-4-hydroxy-D-mandelic acid, a urinary metabolite of norepinephrine', Biochimica et biophysica acta, 25(2), pp. $422-3$.

Copeland, J. and Robertson, H. A. (1982) 'Octopamine as the transmitter at the firefly lantern: Presence of an octopamine-sensitive and a dopamine-sensitive adenylate cyclase', Comparative Biochemistry and Physiology Part C: Comparative Pharmacology. Pergamon, 72(1), pp. 125-127. doi: 10.1016/0306-4492(82)90217-9.

Dimic, D. et al. (2019) 'The reactivity of dopamine precursors and metabolites towards ABTS•: An experimental and theoretical study', Journal of the Serbian Chemical Society, 84, pp. 1-13. doi: 10.2298/JSC190430050D.

Dimić, D. et al. (2017a) 'Antiradical activity of catecholamines and metabolites of dopamine: theoretical and experimental study', Phys. Chem. Chem. Phys. The Royal Society of Chemistry, 128, pp. 16655-16663. doi: 10.1039/C7CP01716B.

Dimić, D. et al. (2017b) 'Antiradical activity of catecholamines and metabolites of dopamine: Theoretical and experimental study', Physical Chemistry Chemical Physics, 19(20). doi: $10.1039 / \mathrm{c} 7 \mathrm{cp} 01716 \mathrm{~b}$.

Dimić, D. et al. (2018) 'Experimental and theoretical elucidation of structural and antioxidant properties of vanillylmandelic acid and its carboxylate anion', Spectrochimica Acta Part A: Molecular and Biomolecular Spectroscopy. Elsevier, 198, pp. 61-70. doi: 10.1016/J.SAA.2018.02.063.

Dimić, D. S. et al. (2017) 'Thermodynamic and kinetic analysis of the reaction between biological catecholamines and chlorinated methylperoxy radicals', Molecular Physics. doi: 10.1080/00268976.2017.1414967.

Dimitrić Marković, J. M. et al. (2014) 'The preferred radical scavenging mechanisms of fisetin and baicalein towards oxygen-centred radicals in polar protic and polar aprotic solvents', RSC Adv., 4(61), pp. 32228-32236. doi: 10.1039/C4RA02577F.

Dunning, T. H. (1989) 'Gaussian basis sets for use in correlated molecular calculations. I. The atoms boron through neon and hydrogen', The Journal of Chemical Physics. AIP Publishing, 90(2), p. 1007. doi: 10.1063/1.456153.

Filipović, M. et al. (2015) 'QSAR of the free radical scavenging potency of selected hydroxybenzoic acids and simple phenolics', Comptes Rendus Chimie, 18(5), pp. 492-498. doi: 10.1016/j.crci.2014.09.001. 
Frisch, M. J. et al. (2009) 'Gaussian 09'. Wallingford CT,: Gaussian, Inc. Available at: http://www.gaussian.com.

Galano, A. et al. (2016) 'Food Antioxidants: Chemical Insights at the Molecular Level', Annual Review of Food Science and Technology. Annual Reviews , 7(1), pp. 335-352. doi: 10.1146/annurev-food-041715-033206.

González, P. M., Aguiar, M. B. and Malanga, G. (2013) 'Electronic paramagnetic resonance (EPR) for the study of ascorbyl radical and lipid radicals in marine organisms', Comparative Biochemistry and Physiology Part A: Molecular \& Integrative Physiology. Pergamon, 165(4), pp. 439-447. doi: 10.1016/J.CBPA.2013.02.021.

Gülçin, İ. (2009) 'Antioxidant activity of 1-adrenaline: A structure-activity insight', ChemicoBiological Interactions, 179(2), pp. 71-80. doi: 10.1016/j.cbi.2008.09.023.

Halliwell, B. and Gutteridge, J. M. C. (1999) Free Radicals in Biology and Medicine. 3rd edn. Oxford: Clarendon Press.

Kladna, A. et al. (2013) 'Superoxide anion radical scavenging property of catecholamines.', Luminescence, 28(4), pp. 450-455. doi: 10.1002/bio.2475.

Kładna, A. et al. (2012) 'Scavenging of hydroxyl radical by catecholamines', Luminescence, 27(6), pp. 473-477. doi: 10.1002/bio.1377.

Laranjinha, J. and Cadenas, E. (2002) 'Oxidation of DOPAC by nitric oxide: effect of superoxide dismutase', Journal of Neurochemistry. Wiley/Blackwell (10.1111), 81(4), pp. 892-900. doi: 10.1046/j.1471-4159.2002.00900.x.

Leopoldini, M., Russo, N. and Toscano, M. (2011) 'The molecular basis of working mechanism of natural polyphenolic antioxidants', Food Chemistry. Elsevier, 125(2), pp. 288-306. doi: 10.1016/J.FOODCHEM.2010.08.012.

Lopes Jesus, A. J. et al. (2015) 'Conformational preferences of 3,4-dihydroxyphenylacetic acid (DOPAC).', Spectrochimica acta. Part A, Molecular and biomolecular spectroscopy, 140, pp. 54-64. doi: 10.1016/j.saa.2014.12.078.

Marenich, A. V., Cramer, C. J. and Truhlar, D. G. (2009) 'Universal Solvation Model Based on Solute Electron Density and on a Continuum Model of the Solvent Defined by the Bulk Dielectric Constant and Atomic Surface Tensions', The Journal of Physical Chemistry B. American Chemical Society, 113(18), pp. 6378-6396. doi: 10.1021/jp810292n.

Marković, Z. et al. (2014) 'Investigation of the radical scavenging potency of hydroxybenzoic acids and their carboxylate anions', Monatshefte für Chemie - Chemical Monthly. Springer Vienna, 145(6), pp. 953-962. doi: 10.1007/s00706-014-1163-3.

Marković, Z. et al. (2015) 'Investigation of the antioxidant and radical scavenging activities of some phenolic Schiff bases with different free radicals.', Journal of molecular modeling, 21(11), p. 293. doi: 10.1007/s00894-015-2840-9.

Marković, Z. et al. (2016) 'Revisiting the solvation enthalpies and free energies of the proton and electron in various solvents', Computational and Theoretical Chemistry, 1077, pp. 1117. doi: 10.1016/j.comptc.2015.09.007.

Milenković, D. et al. (2017) 'Free Radical Scavenging Potency of Dihydroxybenzoic Acids', Journal of Chemistry. Hindawi Publishing Corporation, 2017, pp. 1-9. doi: $10.1155 / 2017 / 5936239$.

Petrović, Z. D. et al. (2015) 'Experimental and theoretical study of antioxidative properties of some salicylaldehyde and vanillic Schiff bases', RSC Adv., 5(31), pp. 24094-24100. doi: 10.1039/C5RA02134K.

Pietri, S. et al. (1990) 'Ascorbyl free radical as a reliable indicator of free-radical-mediated myocardial ischemic and post-ischemic injury. A real-time continuous-flow ESR study', European Journal of Biochemistry. John Wiley \& Sons, Ltd (10.1111), 193(3), pp. 845854. doi: 10.1111/j.1432-1033.1990.tb19408.x. 
Roginsky, V. A. and Stegmann, H. B. (1994) 'Ascorbyl radical as natural indicator of oxidative stress: quantitative regularities.', Free radical biology \& medicine, 17(2), pp. 93-103. Available at: http://www.ncbi.nlm.nih.gov/pubmed/7959177 (Accessed: 10 August 2018).

Samsonowicz, M. et al. (2014) 'Molecular structure and spectroscopic analysis of homovanillic acid and its sodium salt - NMR, FT-IR and DFT studies', Spectrochimica Acta Part A: Molecular and Biomolecular Spectroscopy, 118, pp. 1068-1074. doi: 10.1016/j.saa.2013.09.123.

Sârbu, C. and Casoni, D. (2013) 'Comprehensive evaluation of biogenic amines and related drugs' antiradical activity using reactive 2,2-diphenyl-1-picrylhydrazyl (DPPH) radical', Open Chemistry, 11(5), pp. 679-688. doi: 10.2478/s11532-013-0210-y.

Satoh, K. and Sakagami, H. (1996) 'Ascorbyl radical scavenging activity of polyphenols.', Anticancer research, 16(5A), pp. 2885-90. Available at: http://www.ncbi.nlm.nih.gov/pubmed/8917403 (Accessed: 14 June 2019).

Shimizu, T. et al. (2010) 'Structure Effect on Antioxidant Activity of Catecholamines toward Singlet Oxygen and Other Reactive Oxygen Species in vitro.', Journal of clinical biochemistry and nutrition, 47(3), pp. 181-90. doi: 10.3164/jcbn.09-112.

Sies, H. (1991) Oxidative Stress: Oxidants and Antioxidants, . New York: Academic Press.

Tošović, J. et al. (2017) 'Antioxidative mechanisms in chlorogenic acid', Food Chemistry, 237, pp. 390-398. doi: 10.1016/j.foodchem.2017.05.080.

Zhao, Y. and Truhlar, D. G. (2007) 'The M06 suite of density functionals for main group thermochemistry, thermochemical kinetics, noncovalent interactions, excited states, and transition elements: two new functionals and systematic testing of four M06-class functionals and 12 other function', Theoretical Chemistry Accounts, 120(1-3), pp. 215241. doi: 10.1007/s00214-007-0310-x. 